\title{
Correlação entre os Índices Dopplervelocimétricos da Veia Cava Inferior e Ducto Venoso e a Concentração de Hemoglobina do Cordão em Fetos de Gestantes Isoimunizadas
}

Correlation between the Doppler Indices of Inferior Vena Cava and Ductus Venosus and Fetal Umbilical Cord Blood Concentration of Hemoglobin in Pregnant Women with Isoimmunization

Marcos Roberto Taveira, Antônio Carlos Vieira Cabral, Henrique Vitor Leite

\section{RESUMO}

Objetivo: o objetivo principal desse estudo foi verificar se existe correlação entre os indices dopplervelocimétricos da veia cava inferior e do ducto venoso e a concentração sérica da hemoglobina fetal.

Métodos: estudo transversal e prospectivo, realizado entre janeiro de 1998 e junho de 2001. Foram acompanhadas 31 gestantes isoimunizadas com resultado do teste de Coombs indireto maior que 1:8, que foram submetidas à pesquisa de hemólise fetal. Quando foram indicadas as transfusões intra-uterinas intravasculares, a hemoglobina do cordão foi dosada no início do procedimento. Nos demais casos, a hemoglobina do cordão foi mensurada no momento do parto, sempre por cesariana eletiva. Obteve-se um total de 74 procedimentos estudados, definidos como sendo cada transfusão intra-uterina precedida pela dopplervelocimetria venosa. A mensuração da concentração da hemoglobina fetal foi realizada no Hemocue ${ }^{\mathbb{B}}$ (B-Hemoglobin Photometer Hemocue AB; Angelholm, Sweden), dispositivo usado para a determinação quantitativa de hemoglobina no sangue. A dopplervelocimetria da cava inferior e do ducto venoso foi realizada antecedendo a coleta do sangue fetal, sempre em intervalo de tempo inferior a 24 horas. Os indices dopplervelocimétricos estudados foram o indice de pulsatilidade para veias (IPV), o indice do pico de velocidade para veias (IPVV) e a relação entre o pico de velocidade durante a fase de contração atrial e o pico de velocidade na sistole ventricular (relação CA/SV ou indice de pré-carga), na veia cava inferior, e o IPV, IPVV e a relação entre os picos de velocidade da sístole ventricular e da contração atrial (relação SV/CA), no ducto venoso. Foi realizado estudo de correlação entre a dopplervelocimetria da veia cava inferior e do ducto venoso e a hemoglobina do cordão, pela técnica de regressão linear simples. Realizou-se também estudo de associação entre os indices dopplervelocimétricos do compartimento venoso e a hemoglobina fetal inferior a $10 \mathrm{~g} / \mathrm{dL}$, por meio do teste do $\chi^{2}$.

Resultados: foi efetuado um total de 74 procedimentos. Em 23 desses a hemoglobina fetal era inferior a 7,0 g/dL. Houve correlação negativa significativa entre todos os indices dopplerfluxométricos estudados e a concentração de hemoglobina fetal ( $p<0,05)$, ou seja, os valores mais elevados dos indices dopplervelocimétricos foram observados nos fetos com anemia mais acentuada. Os fetos com nivel de hemoglobina inferior ou igual a $10,0 \mathrm{~g} / \mathrm{dL}$ apresentaram valores dos índices dopplervelocimétricos da cava e do ducto venoso acima do percentil 95 para a idade gestacional.

Conclusões: a dopplervelocimetria da cava inferior $e$ do ducto venoso pode ser utilizada como marcador não invasivo da anemia fetal grave.

PALAVRAS-CHAVE: Dopplervelocimetria. Ducto venoso. Veia cava inferior. Isoimunização Rh.

Centro de Medicina Fetal do Hospital das Clínicas da UFMG.

Correspondência:

Marcos Roberto Taveira

Rua dos Otoni 909, sala 2108 - Bairro Santa Efigênia

30150-270 - Belo Horizonte - MG 


\section{Introdução}

O diagnóstico não invasivo da anemia fetal cresceu em importância diante das evidências de que pode ocorrer agravamento da sensibilização materna quando do uso de procedimentos invasivos como a cordocentese. Isto pode ocorrer devido a hemorragia feto-materna significativa que pode estar presente em até $50 \%$ das pacientes submetidas a punção do cordão umbilical ${ }^{1}$.

Dentre os métodos não invasivos que vêm sendo utilizados destacam-se a cardiotocografia e a dopplervelocimetria, que tem sido mais intensamente estudada com esta finalidade, principalmente por não haver relato de complicações relacionadas com o seu uso, além de revelar informações sobre a hemodinâmica fetal.

A hemólise fetal, se progressiva e acentuada, irá reduzir a viscosidade sangüinea, que tem como conseqüência a queda do hematócrito. Isto irá resultar em estado de fluxo hiperdinâmico, como mecanismo compensatório, que tem por objetivo manter constante o aporte tecidual de oxigênio. Fetos anêmicos apresentam aumento na velocidade média de fluxo sangüíneo, detectada pela dopplervelocimetria das artérias aorta torácica descendente, cerebral média e esplênica ${ }^{2-5}$.

No compartimento venoso, tem sido descrita redução no percentual de fluxo reverso na fase de contração atrial, identificado na dopplervelocimetria da veia cava inferior (VCI). No ducto venoso (DV) ocorre aumento global nas velocidades de fluxo, permanecendo normal, ou mesmo aumentado, o pico diastólico final, durante a fase de contração atrial, neste vaso. Estes achados caracterizam resposta fetal compensatória à anemia, gerando estado hipercinético de fluxo, com redução da viscosidade sangüínea, secundária à hemólise nos fetos anêmicos ${ }^{6-8}$.

Em estágios mais avançados de anemia pode surgir a hidropisia fetal, como conseqüência da disfunção hepática ou da insuficiência cardiaca. Nestas situações, pode ocorrer redução do débito cardiaco, atribuída à falência do coração como bomba, surgindo alterações na dopplervelocimetria da VCI e do DV. Haveria aumento no percentual de fluxo reverso na fase de contração atrial, na VCI, e uma redução no fluxo diastólico final, também na fase de contração atrial, do DV. Estes achados são atribuídos a aumento na pós-carga do ventrículo direito, gerando gradiente pressórico em direção ao átrio correspondente, propagando em direção retrógrada para a VCI e DV, resultando no surgimento de pulsações na veia umbilical. Este último é considerado um estágio terminal da insuficiência cardíaca congestiva, apresentando mortalidade perinatal cinco vezes maior que nos fetos que não apresentam pulsatilidade ao Doppler da veia umbilical ${ }^{9,10}$.

O objetivo deste estudo foi verificar a presença da correlação entre a dopplervelocimetria da VCI e do DV e a concentração sérica de hemoglobina de cordão. Procurou-se avaliar ainda se a dopplervelocimetria da VCI e do DV pode ser utilizada no acompanhamento pré-natal dos fetos anêmicos.

\section{Pacientes e Métodos}

Trata-se de estudo transversal e prospectivo desenvolvido no Centro de Medicina Fetal do Hospital das Clínicas da UFMG (CEMEFE-HC-UFMG) no periodo de janeiro de 1998 a junho de 2001, no qual foram incluídas 31 gestantes com isoimunização materna pelo fator Rh. Foram consideradas sensibilizadas quando apresentavam dois testes de Coombs indireto positivo, independente de sua titulação. Confirmada a sensibilização materna, foi realizado o painel de hemáceas para identificação dos antígenos específicos envolvidos na sensibilização.

As pacientes que apresentaram Coombs indireto maior que 1:8 foram submetidas à pesquisa de hemólise fetal por meio da amniocentese, de acordo com protocolo específico do CEMEFE-HCUFMG. Indica-se pesquisa de hemólise fetal, pela espectrofotometria do líquido amniótico, para todas as gestantes isoimunizadas com Coombs indireto com titulação maior ou igual a 1:8. As transfusões intra-uterinas (TIU) são indicadas, sendo a primeira sempre em idade gestacional inferior a 34 semanas, nos casos com espectrofotometria de líquido amniótico nas zonas $1 \mathrm{~A}$ e $1 \mathrm{~B}$. Nas gestantes com mau passado obstétrico para a isoimunização (hidropisia fetal anterior, transfusões intra-uterinas e natimortos em gestações anteriores), indica-se o tratamento intra-uterino com valores da amniocentese na zona $2 \mathrm{~A}$, na curva de Liley modificada ${ }^{11,12}$.

Foram definidos como critérios de inclusão nesse estudo pacientes $\mathrm{Rh}$ negativas com teste de Coombs indireto maior que 1:8. Foram excluídos os fetos com crescimento intra-uterino restrito, gestações gemelares e, quando presentes, as anomalias estruturais ou doenças cromossômicas.

Adotados estes critérios de inclusão e de exclusão, obteve-se um total de 74 procedimentos. Os casos acompanhados foram submetidos a dois tipos de intervenção para obtenção de sangue fetal: fetos submetidos a TIU por cordocentese tiveram a concentração de hemoglobina determi- 
nada no início do procedimento $(\mathrm{n}=57)$; fetos submetidos à cesariana programada tiveram a concentração de hemoglobina do cordão umbilical determinada no momento do parto, imediatamente após sua ligadura $(\mathrm{n}=17)$. Toda TIU precedida pela dopplervelocimetria da VCI e do DV, em que foi medida a hemoglobina no início do procedimento, e toda coleta de sangue de cordão após a ligadura do funículo foram consideradas um procedimento. Desta forma, uma mesma gestante foi analisada mais de uma vez, conforme o total de tranfusões.

Nos casos de TIU, após a punção da veia umbilical com agulha BD calibre 20 , foi coletado 1 $\mathrm{mL}$ de sangue em seringa de insulina. Quando não houve a necessidade da TIU, imediatamente após o parto, foi realizada a clampagem do cordão umbilical e puncionada a veia umbilical, sendo então colhido $1 \mathrm{~mL}$ de sangue em seringa de insulina. O sangue fetal colhido foi imediatamente e analisado no Hemocue ${ }^{\circledR}$ para determinação do nível de hemoglobina.

A dopplervelocimetria da VCI e do DV foi sempre realizada em intervalo inferior a 24 horas antecedendo a coleta do sangue fetal, seja na transfusão intravascular ou no momento do parto.

A dopplerfluxometria foi realizada por um só examinador, visando reduzir a variabilidade interobservador. Os exames foram todos realizados nos aparelhos Sonoace 8800 (Medson) e também no equipamento Acustic envision, $5500 \mathrm{~S}$ (Dornier), com Doppler colorido e pulsátil e sonda setorial na freqüência de $3,5 \mathrm{mHz}$, utilizando-se filtro acústico de $100 \mathrm{~Hz}$. O cursor foi ajustado para abranger totalmente o diâmetro do vaso insonado.

As pacientes foram colocadas em posição de semi-Fowler, com a cabeceira da cama elevada em 30 graus. Todos os exames foram realizados na ausência de movimentos respiratórios ou de movimentos corporais fetais. A análise dos parâmetros dopplervelocimétricos foi obtida após a observação de cinco ciclos cardiacos consecutivos e com as ondas de velocidade de fluxo (OVF) homogêneas e simétricas. Os índices analisados neste estudo foram mensurados em cada um dos cinco fluxogramas obtidos e calculada a média aritmética.

A VCI foi insonada em qualquer ponto ao longo de seu trajeto, evitando-se apenas a porção mais próxima de sua entrada no átrio direito, em virtude do relato da existência de vestíbulo venoso subdiafragmático, onde não só a VCI, mas também o DV e as veias hepáticas, drenariam seus respectivos fluxos sangüineos, evitando-se assim a interferência nas OVF da VCI. O fluxograma da VCI foi obtido preferencialmente em corte parassagital direito, com o transdutor ligeiramente oblíquo, localizando o vaso de trajeto paralelo à aorta abdominal, dirigindo-se anteriormente em direção ao átrio direito fetal ${ }^{13}$.

O DV foi insonado em seu ponto de origem na veia umbilical. A obtenção das OVF do DV foi realizada preferencialmente em corte longitudinal, parassagital obliquo. Em alguns casos, a dopplervelocimetria do DV foi obtida em corte transverso do abdome fetal, localizando-se a bifurcação da veia umbilical e a origem do vaso por meio do fluxo sangüineo mais brilhante identificado pelo mapeamento colorido, característico desse vaso, pela sua alta velocidade de fluxo ${ }^{14,15}$.

Para a análise dopplervelocimétrica da VCI utilizaram-se o índice de pulsatilidade para veias (IPV), o índice do pico de velocidade para veias (IPVV) e o índice de pré-carga ou relação contração atrial/ sistole ventricular $(\mathrm{CA} / \mathrm{SV})^{10,15-17}$.

Para o estudo dopplerfluxométrico do DV também foram utilizados o IPV e o IPVV, além da relação SV/CA ${ }^{15,16}$.

Para a estimativa amostral utilizou-se o odds ratio médio de 8,5 , calculado no grupo com anemia fetal moderada (hemoglobina do cordão menor que $10,0 \mathrm{~g} / \mathrm{dL}$ ), indicando a chance de se encontrar taxa de hemoglobina fetal menor que 10,0 $\mathrm{g} / \mathrm{dL}$ em fetos com dopplervelocimetria venosa alterada. Esse valor corresponde à média aritmética de todos os valores obtidos de odds ratio para cada um dos índices dopplervelocimétricos estudados no grupo de fetos com hemoglobina inferior a $10,0 \mathrm{~g} / \mathrm{dL}$.

Considerou-se poder do estudo de $80 \%$ (erro beta de $20 \%$ ) e erro alfa (duas caudas) de $5 \%$. Nessa amostra, a relação entre fetos sem anemia para fetos anêmicos foi de $2: 1$ e a freqüência esperada de exame dopplervelocimétrico alterado em fetos sem anemia foi de $10 \%$. O tamanho resultante foi de 54 pacientes, sendo necessário um total de 18 fetos anêmicos e 36 não anêmicos ou com anemia leve. Esse modelo foi utilizado por não se ter conhecimento acerca de investigação clínica prévia com metodologia científica semelhante a este estudo. Para o cálculo da estimativa amostral utilizou-se o programa Epi-Info ${ }^{\circledR}$, versão 6, do ano de 1993.

A análise dos dados foi executada empregando-se o programa MINITAB Inc. ${ }^{\circledR}$, versão 11.12 , de 1996. O nivel de significância aceitável para os testes de hipótese foi de $p<0,05$. A relação de dependência entre as variáveis contínuas IPV e IPVV comuns à VCI e ao $\mathrm{DV}$, a relação $\mathrm{CA} / \mathrm{SV}$ para a $\mathrm{VCI}$ e a relação $\mathrm{SV} / \mathrm{CA}$ para o ducto venoso com a concentração de hemoglobina fetal foram verificadas pela regressão dos mínimos quadrados e análise de variância. A adequação do modelo foi feita pelo teste de hipótese HO: $\beta=0$. 
Foi realizada regressão linear simples univariada para cada um dos índices dopplerfluxométricos estudados e sua relação com a hemoglobina fetal.

Realizou-se também um estudo de associação entre cada uma das variáveis dopplerfluxométricas e a concentração de hemoglobina fetal pelo teste do $\chi^{2}$, quando foram cotejados os dados da dopplervelocimetria do compartimento venoso (normal ou alterado) com a taxa de hemoglobina do cordão (menor ou igual a $10 \mathrm{~g} / \mathrm{dL}$ ou maior que $10,0 \mathrm{~g} / \mathrm{dL}$ ), usando tabelas $2 \times 2$. Considerou-se significativo valor de $\mathrm{p}<0,05$. Quando necessário utilizou-se o teste exato de Fisher. Para essa análise foi utilizado o programa Epi-Info ${ }^{\circledR}$, versão 2000.

As pacientes foram orientadas quanto ao estudo e assinaram termo de consentimento prévio à realização dos procedimentos invasivos e não invasivos. A pesquisa foi aprovada pela Comissão de Ética em Pesquisa do Hospital das Clínicas da UFMG (COEP, HC, UFMG).

\section{Resultados}

Durante o período de realização deste estudo foram acompanhadas 31 gestantes isoimunizadas, de acordo com os critérios de inclusão previamente descritos. Desse total, em 26 pacientes foram realizadas 57 transfusões intravasculares, com média de 2,23 procedimentos por paciente, com mínimo de uma e máximo de seis TIU.

Em 57 situações a hemoglobina fetal foi obtida pela cordocentese, por ocasião das transfusões intravasculares. Nos restantes 17 casos, o sangue fetal foi obtido no momento do parto, logo após a ligadura do cordão umbilical.

Entre esses 74 procedimentos, em 14 $(18,91 \%)$ a concentração de hemoglobina prétransfusional encontrava-se menor ou igual a 7,0 $\mathrm{g} / \mathrm{dL}$, constituindo subgrupo de fetos gravemente anêmicos. Em nove casos (12,16\%), com anemia moderada, o nivel de hemoglobina encontrava-se entre 7 e $10 \mathrm{~g} / \mathrm{dL}$, resultando num total de $23 \mathrm{fe}-$ tos com anemia significativa $(31,08 \%)$. Nos 51 casos restantes, a concentração de hemoglobina fetal era superior a 10,0 g/dL.

A concentração média da hemoglobina do cordão (obtida no momento da realização da cordocentese ou colhida por ocasião do parto) variou entre 3,3 e 17,3 g/dL, com média de $11,2 \mathrm{~g} \%$.

O IPV, na VCI, apresentou os seguintes valores: média de 2,92 , mediana de 2,7 , valor mínimo de 1,71 e máximo de 10,1 , com um desvio-pa- drão de $1,03,1^{\circ}$ quartil de 2,48 e $3^{\circ}$ quartil de 3,04 .

O IPVV, na VCI, apresentou a seguinte variabilidade: média $=2,24$, mediana $=2,7$, valor minimo de 1,31 e máximo de 7,8, desvio-padrão de 0,95 e valor de $1^{\circ}$ quartil $=1,73$ e $3^{\circ}$ quartil $=2,36$.

A relação CA/SV na VCI apresentou os seguintes valores: média $=0,32$, mediana $=0,29$, mínimo de 0,13 , máximo de 0,84 , desvio-padrão de $0,12,1^{\circ}$ quartil $=0,24$ e $3^{\circ}$ quartil $=0,35$.

O IPV do DV, apresentou a seguinte variabilidade: média $=0,75$, mediana $=0,69$, mínimo $=$ 0,39 , máximo $=1,86$, desvio-padrão $=0,63,1^{\circ}$ quartil $=0,58$ e $3^{\circ}$ quartil $=0,87$.

O IPVV do DV apresentou os seguintes valores: média $=0,68$, mediana $=0,64$, mínimo $=0,38$, máximo $=1,28$, desvio-padrão de $0,21,1^{\circ}$ quartil $=$ 0,52 e $3^{\circ}$ quartil $=0,79$.

A relação SV/CA no DV apresentou a seguinte variabilidade: média $=2,25$, mediana $=2,10$, mínimo $=1,45$, máximo $=4,0$, desvio-padrão $=2,66$, $1^{\circ}$ quartil $=1,82$ e $3^{\circ}$ quartil $=2,47$.

Verificou-se, neste estudo, que houve associação significativa entre a dopplervelocimetria da VCI e do DV alteradas com a hemoglobina do cordão menor ou igual a $10 \mathrm{~g} / \mathrm{dL}$. Os conceptos com hemoglobina do cordão menor ou igual a $10,0 \mathrm{~g} / \mathrm{dL}$ apresentaram, em sua maioria, valores dopplervelocimétricos da VCI e do DV acima do percentil 95 para a respectiva idade gestacional, sendo que todos esses parâmetros citados apresentaram valores de $p<0,05$. Estes resultados estão discriminados nas Tabelas 1,2 e 3 e indicam que a dopplervelocimetria da VCI e do DV, independente do índice escolhido para seu estudo, estará alterada predominantemente no grupo de fetos com concentração da hemoglobina do cordão menor ou igual a $10 \mathrm{~g} / \mathrm{dL}$.

Analisou-se o grupo estudado como um todo, compreendendo, portanto, fetos anêmicos e não anêmicos, no intuito de verificar e quantificar a existência de correlação estatística entre as variáveis dopplervelocimétricas da VCI e do DV e a concentração de hemoglobina determinada no sangue fetal.

Equações foram obtidas pela metodologia da análise estatística de regressão linear simples, considerando-se o efeito da concentração da hemoglobina do cordão (variável preditora) sobre cada um dos índices dopplerfluxométricos estudados (variável resposta). Observou-se a existência de uma relação de dependência negativa entre os índices dopplerluxométricos e a taxa de hemoglobina fetal. 
Tabela 1 - Associação entre os índices dopplervelocimétricos do ducto venoso (IPV, IPVV e relação SV/CA) e a concentração de hemoglobina do cordão inferior ou igual a $10 \mathrm{~g} /$ $\mathrm{dL}$ em fetos de gestantes isoimunizadas.

\begin{tabular}{|c|c|c|c|}
\hline & $\mathrm{Hb} \leq 10 \mathrm{~g} \%$ & $\mathrm{Hb}>10 \mathrm{~g} \%$ & Total \\
\hline IPV alterado & 11 & 6 & 17 \\
\hline IPV normal & 12 & 45 & 57 \\
\hline IPVV alterado & 12 & 11 & 23 \\
\hline IPVV normal & 11 & 40 & 51 \\
\hline Relação SV/CA alterada & 9 & 2 & 11 \\
\hline Relação SV/CA normal & 14 & 49 & 51 \\
\hline $\begin{array}{l}\text { IPV } \\
\chi^{2}=11,65, p<0,001\end{array}$ & $\begin{array}{l}\text { IPW } \\
\chi^{2}=6,93, p<0,001\end{array}$ & \multirow{2}{*}{\multicolumn{2}{|c|}{$\begin{array}{c}\text { Relação SV/CA } \\
\chi^{2}=15,53, p<0,01 \\
\text { Teste exato de Fisher } p \\
S=39,7 \% \\
E=96,1 \% \\
\text { VPP }=81,81 \% \\
\text { VPN }=77,8 \%\end{array}$}} \\
\hline $\begin{array}{l}S=47,8 \% \\
E=88,2 \% \\
V P P=64,7 \% \\
V P N=78,9 \%\end{array}$ & $\begin{array}{l}S=52,1 \% \\
E=78,4 \% \\
V P P=52,2 \% \\
V P N=78,4 \%\end{array}$ & & \\
\hline
\end{tabular}

$\chi^{2}=$ qui-quadrado; $\mathrm{S}=$ sensibilidade; $\mathrm{E}=$ especificidade; VPP = valor preditivo positivo; VPN = valor preditivo negativo; IPV - índice de pulsatilidade para veias; IPVV - índice do pico de velocidade para veias; Relação SV/CA - relação entre o pico de velocidade na sístole ventricular/pico de velocidade na contração atrial.
Tabela 2 - Associação entre os índices dopplervelocimétricos da veia cava inferior (IPV, IPVV e relação CA/SV) e a concentração de hemoglobina do cordão igual ou inferior a $10,0 \mathrm{~g} / \mathrm{dL}$, em fetos de gestantes isoimunizadas.

\begin{tabular}{|c|c|c|c|}
\hline & $\mathrm{Hb} \leq 10 \mathrm{~g} \%$ & $\mathrm{Hb}>10 \mathrm{~g} \%$ & Total \\
\hline IPV alterado & 11 & 03 & 14 \\
\hline IPV normal & 12 & 48 & 60 \\
\hline IPVV alterado & 12 & 11 & 23 \\
\hline IPVV normal & 11 & 40 & 51 \\
\hline Relação CA/SV alterada & 12 & 05 & 17 \\
\hline Relação CA/SV normal & 11 & 46 & 57 \\
\hline $\begin{array}{l}\text { IPV } \\
\chi^{2}=18,18, p<0,001\end{array}$ & $\begin{array}{l}\text { IPW } \\
\chi^{2}=6,93, p<0,001\end{array}$ & \multirow{2}{*}{$\begin{aligned} & \text { Relação CA/S } \\
& \chi^{2}=16,08, p< \\
& \text { Teste exato de Fisch } \\
& \text { S }=52,2 \% \\
& \text { E }=90,2 \% \\
& \text { VPP }=70,6 \% \\
& \text { VPN }=80,7 \%\end{aligned}$} & $\begin{array}{l}1 \\
0<0,001\end{array}$ \\
\hline $\begin{array}{l}S=47,8 \% \\
E=94,1 \% \\
V P P=78,6 \% \\
V P N=80 \%\end{array}$ & $\begin{array}{l}S=52,2 \% \\
E=78,4 \% \\
V P P=52,2 \% \\
V P N=78,4 \%\end{array}$ & & \\
\hline
\end{tabular}

$\chi^{2}=$ qui-quadrado; $S$ = sensibilidade; $E$ = especificidade; VPP = valor preditivo positivo; VPN = valor preditivo negativo; IPV = índice de pulsatilidade para veias; IPVV = índice do pico de velocidade para veias; Relação CA/SV (índice de pré-carga) - relação entre 0 pico de velocidade na contração atrial e o pico de velocidade na sístole ventricular; $\mathrm{VCl}$ - veia cava inferior

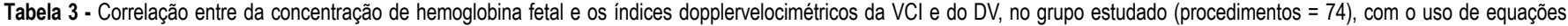
de regressão linear.

\begin{tabular}{|c|c|c|c|c|c|c|c|c|c|}
\hline $\begin{array}{l}\text { Índice } \\
\text { dopplervelocimétrico }\end{array}$ & $\begin{array}{l}\text { Coeficiente } \\
\text { Constante }\end{array}$ & $\begin{array}{c}\text { DP da } \\
\text { constante }\end{array}$ & $\begin{array}{c}\text { Coeficiente } \\
\mathrm{Hb}\end{array}$ & $\begin{array}{l}\mathrm{DP} \\
\mathrm{Hb}\end{array}$ & $\begin{array}{l}\text { Graus de } \\
\text { liberdade }\end{array}$ & SS & $\mathbf{F}$ & $p$ & Equação da regressão \\
\hline IPV - ducto venoso & 0,97 & 0,10 & $-0,19$ & 0,008 & 1 & 0,34 & 5,08 & $p=0,027$ & PV- DV $=0,97-0,019 \mathrm{Hb}$ \\
\hline IPVV - ducto venoso & 0,84 & 0,08 & $-0,014$ & 0,00 & 1 & 0,19 & 4,7 & $p=0,032$ & IPVV - DV $=0,84-0,0145 \mathrm{Hb}$ \\
\hline $\begin{array}{c}\text { Relação SV/CA } \\
\text { ducto Venoso }\end{array}$ & 2,98 & 0,22 & $-0,06$ & 0,01 & 1 & 3,89 & 11,3 & $p=0,001$ & SV/CA- DV $=2,99-0,065 \mathrm{Hb}$ \\
\hline IPV - VCl & 4,28 & 0,36 & $-0,12$ & 0,03 & 1 & 13,5 & 15,0 & $p<0,001$ & IPV- DV $=4,28-0,121 \mathrm{Hb}$ \\
\hline IPVV - VCl & 3,7 & 0,31 & $-0,13$ & 0,02 & 1 & 15,6 & 22,8 & $p<0,001$ & IPVV- DV= 3,7 $-0,13 \mathrm{Hb}$ \\
\hline Relação CA/SV & 0,51 & 0,043 & $-0,01$ & 0,003 & 1 & 0,25 & 20,2 & $p<0,001$ & CA/SV $-D V=0,51-0,016 \mathrm{Hb}$ \\
\hline
\end{tabular}

F: parâmetro de Fisher para o teste de significância; $S S$ = soma dos mínimos quadrados; $p$ = nível de significância

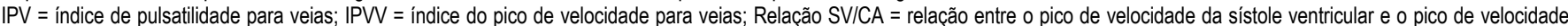
da contração atrial; Relação CA/SV = relação entre o pico de velocidade da contração atrial e o pico de velocidade da sístole ventricular; $\mathrm{VCl}=$ veia cava inferior

$\mathrm{DV}=$ ducto venoso; $\mathrm{Hb}=$ hemoglobina $; \mathrm{DP}=$ desvio-padrão.

\section{Discussão}

A normatização da cordocentese no tratamento da anemia fetal principalmente resultante dos quadros de isoimunização materna pelo fator Rh foi o principal fator responsável pelo aumento da sobrevida perinatal, sobretudo nos fetos hidrópicos. Entretanto, a punção do cordão umbilical é procedimentro potencialmente perigoso, podendo ocorrer hemorragia feto-materna em até $50 \%$ das vezes. A taxa de perda fetal relacionada ao procedimento varia entre 1,5 e $5 \%$, sendo superior à da amniocentese $\mathrm{e}^{1,12}$.

O diagnóstico não invasivo da anemia fetal passou a ser uma necessidade da medicina fetal, em virtudes das possiveis complicações da cordocentese, reservando este procedimento apenas para os casos mais graves, que necessitem da terapia intra-uterina.

A dopplervelocimetria tem sido o método mais pesquisado, pois além de ser método não invasivo, fornece informações importantes sobre a hemodinâmica fetal, sendo capaz de detectar as repercussões circulatórias sobre o concepto.

Desta forma, tem sido descrito estado hiperdinâmico de fluxo, em resposta à anemia fetal, visando compensar a queda de viscosidade sangüinea e do hematócrito, secundária à hemólise. Nestes casos há aumento no débito car- 
diaco. Este achado já foi detectado e caracterizado por aumento na velocidade média de fluxo nas artérias aorta torácica descendente, cerebral média e esplênica. No compartimento venoso, observa-se diminuição no percentual de fluxo reverso durante a fase de contração atrial, na dopplervelocimetria da VCI. No DV, existe aumento nas velocidades de fluxo, sendo que o pico diastólico final, também durante a sístole atrial, pode estar normal ou mesmo aumentado ${ }^{3,5,7-9}$.

Os melhores resultados no diagnóstico não invasivo da anemia fetal são obtidos com a medida do pico de velocidade sistólica na artéria cerebral média, com sensibilidade superior a $90 \%$, que está aumentado nos fetos anêmicos, refletindo aumento no débito cardiaco, para compensar a queda da hemoglobina ${ }^{18-20}$. Sabe-se que nos casos mais graves, os fetos podem apresentar algum grau de disfunção cardíaca, podendo chegar até mesmo a um quadro de insuficiência cardiaca congestiva.

A dopplervelocimetria do compartimento venoso fornece informações precisas sobre a função ventricular direita. Qualquer situação em que ocorra aumento na pós-carga do ventrículo direito, ou que leve a alterações no padrão de enchimento ventricular, irá originar gradiente pressórico entre ventrículo e átrio direitos, causando aumento de pressão retrógrada em direção a cava inferior, resultando em aumento do percentual de fluxo reverso durante a fase de contração atrial. Se o processo não for interrompido, atingirá também o ducto venoso, onde haverá redução no pico de velocidade de fluxo ao final da diástole, também na sístole atrial, que em casos extremos poderá estar ausente ou mesmo reverso. A resultante desse processo será o surgimento de pulsatilidade na veia umbilical, indicando a existência de quadro de $\operatorname{ICC}^{10,14,15}$.

A opção pela utilização desses índices dopplerfluxométricos deveu-se a três motivos: são independentes do ângulo de insonação, não sendo, portanto, necessária a sua correção; todos os três índices são de fácil obtenção; estão entre os mais utilizados pelos relatos da literatura ${ }^{9,13-15}$.

O estudo dopplervelocimétrico do compartimento venoso pode ser útil no acompanhamento de fetos anêmicos, transfundidos ou não, detectando casos onde já exista algum grau de comprometimento da função cardiaca. Nestes casos a dopplervelocimetria pode indicar a antecipação de um novo procedimento invasivo, amniocentese ou transfusão intra-uterina, de acordo com o resultado do exame. Pode ainda indicar a necessidade de se realizarem transfusões intra-uterinas de menor volume e em intervalos menores, nos ca- sos mais graves, evitando-se assim o risco de sobrecarga de volume, que poderia ser fatal.

Em nosso estudo, observamos que houve correlação significativa entre as alterações dopplervelocimétricas da VCI e do DV e os menores valores da concentração de hemoglobina fetal, para todos os indices estudados. Observamos ainda que fetos com concentração de hemoglobina menor ou igual a $10 \mathrm{~g} / \mathrm{dL}$ apresentavam aumento no percentual de fluxo reverso na VCI e diminuição no fluxo diastólico final no DV, durante a fase de contração atrial de ambos, com correlação significativa para todos os indices estudados. Estes achados indicam comprometimento da função cardiaca, denotando casos de maior gravidade que em algumas vezes não foram detectados em outros exames.

De acordo com os resultados obtidos neste estudo, a dopplervelocimetria da VCI e do DV deve ser utilizada no acompanhamento pré-natal de fetos anêmicos, em conjunto com os demais métodos já utilizados. O exame dopplervelocimétrico do compartimento venoso, ao que parece, não apresenta sensibilidade suficientemente alta para que possa ser utilizado no diagnóstico da anemia fetal, porém parece ser importante indicador de gravidade da anemia, devendo ser utilizado rotineiramente no acompanhamento dos fetos anêmicos. Desta forma pode-se racionalizar ainda mais a indicação da transfusão intra-uterina, sendo sempre antecipada diante de um exame alterado ou postergada quando estiver normal.

Diante dos resultados aqui obtidos pode-se concluir que a dopplervelocimetria da VCI e do DV pode ser utilizada no acompanhamento pré-natal de fetos anêmicos, sendo importante marcador de anemia fetal grave, quando alterada, indicando haver algum grau de comprometimento da função cardíaca.

\section{ABSTRACT}

Purpose: to determine the relationship between the Doppler indices of inferior vena cava and ductus venosus and the fetal hemoglobin concentration.

Methods: a cross-sectional prospective study was performed at the "Centro de Medicina Fetal HC UFMG" from January 1998 to July 2001. Thirty-one pregnant women with isoimmunization, detected by an indirect Coombs test $>1: 8$, underwent a protocol for the identification of fetal hemolysis. When intrauterine transfusions were indicated, the umbilical cord hemoglobin concentration was measured at the begining of the procedure. In the other cases, it was measured at delivery. Every single intrauterine transfusion preceded by Doppler flow velocity waveforms from inferior vena cava 
and ductus venosus was defined as one case. Hemocue ${ }^{\circledR}(B-$ Hemoglobin Photometer Hemocue AB; Angelholm, Sweden) was used to measure the fetal hemoglobin concentration. In all cases, inferior vena cava and ductus venosus Doppler examinations were performed before the collection of fetal blood samples. For the inferior vena cava Doppler, the studied indices were pulsatility index for veins (PVI), peak velocity index for veins $(P V I V)$ and atrial/systole ratio $(C A / S V$ ratio or preload index); for ductus venosus, PVI, PVIV and systolel atrial ratio ( $S V / C A$ ratio). The relationship between inferior vena cava and ductus venosus Doppler indices and cord blood hemoglobin concentration was obtained by simple linear regression analysis. Moreover, an association between those indices and the finding of fetal hemoglobin $<10 \mathrm{~g} / \mathrm{dL}$ was shown by the $\chi^{2}$ test, significant at $p<0.05$.

Results: seventy-four procedures were studied. In twenty-three cases fetal hemoglobin was below $7 \mathrm{~g} / \mathrm{dL}$. A significant negative correlation between all studied Doppler indices and fetal concentration of hemoglobin was observed $(p<0.05)$. The highest Doppler index values were observed in severe anemic fetuses. Fetuses with cord blood hemoglobin below $10 \mathrm{~g} / \mathrm{dL}$ presented inferior vena cava and ductus venosus Doppler indices over the 95 percentile for gestational age.

Conclusions: Doppler flow velocity waveforms from inferior vena cava and ductus venosus may be used as a noninvasive marker of severe fetal anemia.

KEYWORDS: Venous Doppler. Ductus venosus. Inferior vena cava. Rhesus isoimmunization.

\section{Referências}

1. Bowman JM, Pollock JM, Peterson LE, Harmam CR, Manning FA, Menticoglou SM. Fetomaternal hemorrhage following funipuncture: increase in severity of maternal red-cell alloimmunization. Obstet Gynecol 1994; 84:839-43.

2. Rightmire DA, Nicolaides $\mathrm{KH}$, Rodeck $\mathrm{CH}$, Campbell $\mathrm{S}$. Fetal blood velocities in $\mathrm{Rh}$ isoimmunization: relationship to gestational age and to fetal hematocrit. Am J Obstet Gynecol 1986; 68:233-6.

3. Nicolaides KH, Bilardo KM, Campbell S. Prediction of fetal anemia by measurement of the mean blood velocity in the fetal aorta. Am J Obstet Gynecol 1990; 162:209-12.

4. Mari G, Moise KJ Jr, Deter RL, Kirshon B, Stefos T, Carpenter RJ Jr. Flow velocity waveforms of the vascular system in the anemic fetus before and after intravascular transfusion for severe red-blood cell alloimmunization. Am J Obstet Gynecol 1990; 162:1060-4.

5. Bahado-Singh R, Oz U, Deren O, et al. A new splenic artery Doppler velocimetric index for prediction of severe fetal anemia associated with $\mathrm{Rh}$ alloimmunization. Am J Obstet Gynecol 1999; 180:49-54.

6. Oepkes D, Vandenbussche FP, Van Bel F, Kanhai $\mathrm{HH}$. Fetal ductus venosus blood flow velocities before and after transfusion in red-cell alloimmunized pregnancies. Obstet Gynecol 1993; 82:237-41.

7. Hecher K, Snijders R, Campbell S, Nicolaides KH. Fetal venous, arterial, and intracardiac blood flows in red blood cell isoimmunization. Obstet Gynecol 1995; 85:122-8.

8. Rizzo G, Arduini D, Romanini C. Fetal cardiac function in anemia. In: Arduini D, Rizzo G, Romanini C, editors. Fetal Cardiac Function. $1^{\text {st }}$ ed. New York: Parthenon; 1995. p.103-7.

9. Hecher K, Campbell S. Characteristics of fetal venous blood flow under normal circumstances and during fetal disease. Ultrasound Obstet Gynecol 1996; 7:68-83.

10.Rizzo G, Capponi A, Arduini D, Romanini C. Ductus venosus velocity waveforms in appropriate and small for gestational age fetuses. Early Hum Dev 1994; 39:15-26.

11.Cabral ACV. Isoimunização materna. In: Cabral ACV, editor. Obstetrícia. $2^{a}$ ed. Rio de Janeiro: Revinter; 2002. p.203-9.

12. Cabral ACV, Taveira MR, Lopes APBM, Pereira AK, Leite HV. Transfusão intra-uterina na isoimunização materna pelo fator Rh. Rev Bras Ginecol Obstet 2001; 23:299-303.

13.Huisman TW, Gittenberger-de Groot AC, Wladimiroff JW. Recognition of a fetal subdiaphragmatic venous vestibulum essential for fetal venous Doppler assessment. Pediatr Res 1992; 32:338-41.

14.Kiserud T, Eik-Nes SH, Blaas HG, Hellevik LR. Ultrasonographic velocimetry of the fetal ductus venosus. Lancet 1991; 338:1412-4.

15.Kiserud T, Eik-Nes SH. The fetal ductus venosus. In: Copel JA, Reed KL, editors. Doppler Ultrasound in Obstetrics and Gynecology. $1^{\text {st }}$ ed. New York: Raven Press; 1995. p.297-305.

16.Hecher K, Campbell S, Snijders R, Nicolaides KH. Reference ranges for fetal venous and atrioventricular blood flow parameters. Ultrasound Obstet Gynecol 1994; 4:381-90.

17.Kanzaki T, Chiba Y. Evaluation of the preload condition of the fetus by inferior vena caval blood flow pattern. Fetal Diagn Ther 1990; 5:168-74.

18. Mari G, Deter RL, Carpenter RL, et al. Noninvasive diagnosis by Doppler ultrasonography of fetal anemia due to maternal red-cell alloimmunization. Collaborative Group for Doppler Assessment of the Blood Velocity in Anemic Fetuses. N Engl J Med 2000; 342:9-14.

19.Abdel-Fattah SA, Soothill PW, Carroll SG, Kyle PM. Noninvasive diagnosis of anemia in hydrops fetalis with the use of middle cerebral artery Doppler velocity. Am J Obstet Gynecol 2001; 185:1411-5.

20.Detti L, Mari G, Akiyama M, et al. Longitudinal assessment of the middle cerebral artery peak systolic velocity in healthy fetuses and in fetuses at risk for anemia. Am J Obstet Gynecol 2002; 187:937-9. 